\title{
Virtual Environment for 3-D Synthetic Fingerprints
}

\author{
Ruggero Donida Labati, Angelo Genovese, Vincenzo Piuri, Fabio Scotti \\ Department of Information Technology \\ Università degli Studi di Milano \\ Milano, 20122, Italy. \\ \{ruggero.donida, angelo.genovese, vincenzo.piuri, fabio.scotti\}@unimi.it
}

\begin{abstract}
Contactless biometric recognition performed using three-dimensional fingerprint models has the advantages of reducing problems related to the deformations of the skin, dust on the sensor, and spoofing of latent fingerprints. Moreover, the fingerprint area usable for the recognition is wider than the one captured by traditional contact-based acquisition techniques.

The design of fingerprint recognition systems based on threedimensional models, however, requires the collection of large datasets of samples. This task is expensive and time consuming, and can also require the implementation of new hardware setups. For this reason, the use of virtual environments for the computation of realistic synthetic models can be useful for the evaluation of new biometric algorithms.

In the literature, there are methods for the computation of synthetic fingerprint models that resemble fingerprint images acquired using a touch-based sensor. However, at the best of our knowledge, there are no works dealing with the computation of synthetic three-dimensional models of fingerprints.

In this paper, we propose a virtual environment able to compute synthetic three-dimensional fingerprints, which can be useful for the entire research community working in the fields of biometrics and three-dimensional reconstruction.

The method is based on image processing techniques and algorithms designed for fingerprint recognition applications. The method is validated by comparing the results obtained with contactless fingerprint acquisitions. Results show that the method is feasible and produces three-dimensional models that are realistic and visually comparable with real fingerprints.
\end{abstract}

Index Terms-virtual environment, fingerprint, threedimensional, contactless.

\section{INTRODUCTION}

Biometric systems that use three-dimensional fingerprint models can overcome some of the major limitations of traditional contact-based sensors, such as the presence of deformations in the captured images due to different pressures of the finger on the sensor, the presence of dirt on the sensor surface due to unclean hands, and the possibility to obtain latent fingerprints. Moreover, the use of the information obtained by computing a complete three-dimensional reconstruction of the fingerprint can increase the accuracy of the biometric recognition. The three-dimensional fingerprint models, in fact, permit to use a wider area of the ridge pattern, and to use new features related to the three-dimensional shape of the ridges.

The use of standard CCD cameras for the contactless recognition of fingerprints is currently being researched. However, the images captured by CCD cameras are very different from the ones obtained using contact-based sensors. Contactless images, in fact, can present problems related to the lower contrast between the ridges and valleys, reflections, and a more (a)

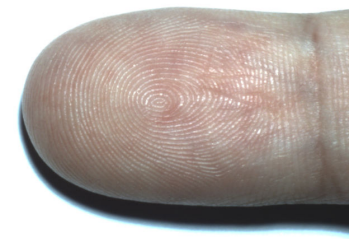

(c)

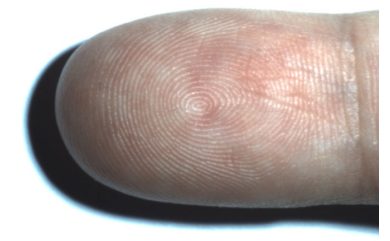

(b)

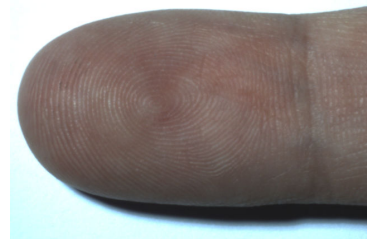

(d)

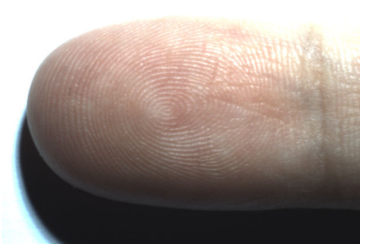

Fig. 1. Fingerprints captured contactless with different illuminations: (a) frontal illumination; (b) illumination from the left side; (c) illumination from the right side; (d) illumination from the top side.

complex background. Even the skin, in fact, can be considered as a part of the background. Moreover, the quality of the obtained images is strictly related to the used illumination technique. Examples of contactless fingerprints captured using a led light placed in different positions are shown in Fig. 1. The images shown in Fig. 1a, Fig. 1b, Fig. 1c, Fig. 1d were captured with a frontal illumination, illumination from the left side, illumination from the right side, and illumination from the top side, respectively. It is possible to observe that the image shown in Fig. 1a has the most wide usable area, while the other fingerprint images present different kinds of problems, such as, underexposure (Fig. 1b), shadows (Fig. 1c), and reflections (Fig. 1d).

In the literature, there are different methods designed for the computation of three-dimensional fingerprint models based on contactless acquisition techniques [1-4]. However, at the best of our knowledge, no public datasets of contactless fingerprint acquisitions are available.

The design of fingerprint recognition systems based on three-dimensional models requires the collection of sample databases large enough to prove the validity of the envisioned method. This task is expensive and time consuming. Moreover, it can require the design and implementation of new acquisition setups. In order to limit the effort necessary to collect biometric data, it can be useful to perform tests on simulated data. In the literature, some works have dealt with the creation of synthetic fingerprint images that realistically 
resemble the ones acquired using contact-based sensors [510]. However, at the best of our knowledge, there are no works that deal with the computation of synthetic three-dimensional fingerprints. Our method could then be a useful tool for research groups operating in biometrics and three-dimensional reconstruction techniques, allowing to easily simulate different acquisition setups. Moreover, the proposed method can permit to compare the results of future algorithms with a ground truth of simulated samples.

Virtual environments for the simulation of physical situations, designed with the purpose of testing computer vision techniques, have been proposed in the literature for different applicative contexts and obtained positive results [11,12].

In this paper, we propose a virtual environment for the computation of synthetic three-dimensional models of fingerprints. The proposed method permits to simulate contactless fingerprint acquisitions using fingerprint images captured by contact-based sensors. The method is designed to simulate images captured with different kinds of cameras and in different illumination conditions. Image processing techniques and well-known algorithms designed for contact-based fingerprint recognition systems are used to compute the synthetic threedimensional models. First, the information related to the ridge pattern is estimated from the contact-based fingerprint image. Then, perturbations are applied on the obtained data in order to simulate a realistic ridge pattern. The three-dimensional structure of the ridges is then computed by considering the average height of the human ridges. The model is completed with the superimposition of the obtained three-dimensional ridges on a parametric shape representing the central region of the finger. The next step is the simulation of the blur introduced by optics in real contactless acquisitions. Realistic colors and proper illumination conditions are finally applied in order to increase the realism of the model.

The paper is structured in the following way: in Section II a literature review is presented, and in Section III the proposed method is discussed. Section IV contains the experimental results, and Section V summarizes the paper.

\section{PREVIOUS WORK}

In the literature, several methods have been proposed in order to compute three-dimensional fingerprint models [1-4]. The method proposed in [1] is designed to compute a threedimensional model of the fingerprint by using an illuminator shaped as a ring-mirror. A camera captures circular portions of the finger, which are then merged in the three-dimensional model. A technique based on a shape from silhouette approach and multiple view algorithms is described in [3]. The method uses five cameras placed around the finger to compute its volume, which is then refined using the corresponding points in the image pairs. A method based on a structured light technique is described in [3], and is based on the projection of a pattern computed from a sinusoidal wave. The method proposed in [4] computes a three-dimensional reconstruction of the fingerprint using a two-view acquisition system and a static projected pattern.
At the best of our knowledge, there are no works concerning the computation of synthetic three-dimensional models of fingerprints. The only works in the field of synthetic fingerprint computation are based on the simulation of two-dimensional images of fingerprints captured by contact-based sensors [5$10]$.

The method proposed in [5] is based on techniques for the computation of global and local features of the fingerprint. The global features are related to the orientation of the ridge pattern, which is modeled by using a limited set of coefficients describing a sampling of the two-dimensional function that represents the spatial direction of the ridges. A second-order model is then used to approximate the spatial orientation function. Local features are computed by first defining a set of points with a binary mask, which is then iteratively refined using a stripe-shaped filter. The orientation of the filter is chosen according to the orientation field.

The method described in [6,7] proposes a biologicallyinspired mathematical model for the formation of the fingerprint ridge pattern, based on the idea that the ridge patterns are created by the differential growth of the skin basal layer, which is compressed by the adjacent skin layers. The compression that originates the fingerprint pattern is then modeled and reproduced to compute the synthetic fingerprints.

An approach based on a genetic algorithm is introduced in [8]. This approach is designed for the computation of synthetic fingerprint samples from a limited set of real fingerprint images. The genetic algorithm is used to initialize and adapt a set of filters used in order to obtain the synthetic samples. The method is also able to adapt the process in order to produce synthetic samples which are similar to the ones appertaining to a specific set of real fingerprints.

The method described in [9] is based on the computation of different images describing the fingerprint area, the ridge orientation, the ridge frequency, and the ridge pattern. The fingerprint area is defined using a silhouette, while the orientation image is obtained starting from the position of the singular points (loops and deltas). A mathematical flow model is then applied to estimate a consistent orientation in the rest of the image. The frequency information image is obtained by using an heuristic criteria inferred from the visual inspection of real fingerprints. The ridge pattern and the minutiae are then computed by using a contextual iterative Gabor filtering technique. As a last step, the realism of the model is improved by simulating the displacement, rotation, distortion, skin condition, and acquisition noise.

An improved version of the method described in [9] is proposed in [10], and allows also the computation of synthetic fingerprints according to parameters such as gender, age, and race. The possibility to explicitly tune these parameters permits the creation of a database of fingerprints that realistically models the fingerprint distribution related to the applicative context.

\section{THE PROPOSED APPROACH}

The proposed method is designed to simulate synthetic three-dimensional models of fingerprints, resembling contact- 
less fingerprint images captured with different cameras and illumination conditions, using image processing techniques and well-known algorithms designed for fingerprint recognition systems. It focuses on modeling the central region of the fingerprint, since it is the region that contains the most discriminative part of the fingerprint information. The method takes in input a fingerprint image captured using a contact-based sensor, or obtained by applying techniques in the literature for the computation of synthetic fingerprint images. The information related to the ridge pattern is used to compute a realistic three-dimensional model of the fingerprint. Realistic focus, color, and illumination details are then applied in order to increase the quality of the synthetic three-dimensional model.

The method can be divided in the following steps:

1) image preprocessing;

2) ridge pattern analysis;

3) noise injection;

4) computation of the three-dimensional ridges;

5) finger shape simulation;

6) simulation of the lens focus;

7) simulation of the color pattern;

8) simulation of the light source.

An outline of the proposed method is shown in Fig. 2

\section{A. Image preprocessing}

In the preprocessing step, the region of the contact-based fingerprint image describing the most discriminative area of the ridge pattern is selected, then the image is normalized in order to enhance the visibility of the ridge pattern.

The used contact-based fingerprint images are acquired at a resolution of 500 ppi. The images are cropped and a central area of $100 \times 100$ pixels, corresponding to an area of $5 \times 5 \mathrm{~mm}$, is extracted from the image.

The intensity of the cropped image $I$ is then normalized in order to obtain an image with 0 mean and standard deviation equal to 1 .

\section{B. Ridge pattern analysis}

The analysis of the ridge pattern is performed in order to estimate a map image that represents the height of the ridges in a non-dimensional space. The used method assumes that the height of the ridge pattern is proportional to the result obtained by applying tuned Gabor filters to the contact-based fingerprint image. The use of Gabor filters permits to reduce the noise and to obtain ridges with smooth shapes.

We used a well-known method in the literature for the enhancement of contact-based fingerprint images [13]. The first step is the computation of two images that describe the local orientation $O_{r}$ and the local frequency $F_{r}$ of the ridges. Then, a set of Gabor filters tuned by using the estimated information are convolved to the fingerprint image. The evensymmetric Gabor filter has the general form

$$
h(x, y: \phi, f)=\exp \left\{-\frac{1}{2}\left[\frac{x_{\phi}^{2}}{\sigma_{x}^{2}}+\frac{y_{\phi}^{2}}{\sigma_{y}^{2}}\right]\right\} \cos \left(2 \pi f x_{\phi}\right),
$$

$$
\begin{gathered}
x_{\phi}=x \cos (\phi)+y \sin (\phi) \\
y_{\phi}=-x \cos (\phi)+y \sin (\phi)
\end{gathered}
$$

where $\phi$ is the orientation of the Gabor filter, $f$ is the frequency of a sinusoidal plane wave, and $\sigma_{x}$ and $\sigma_{y}$ are the space constants of the Gaussian envelope along the $x$ and $y$ axes, respectively. The parameters $\phi$ and $f$ of the Gabor filter applied to the pixel $(x, y)$ of the image $I$ are selected accordingly to $O_{r}(x, y)$ and $F_{r}(x, y)$ respectively, obtaining the image $G$. The parameters $\sigma_{x}$ and $\sigma_{y}$ are empirically tuned.

In order to search the regions of the image $G$ representing the ridges, a binary image $B$ is computed as :

$$
B(x, y)= \begin{cases}0 & \text { if } G(x, y)<t_{b} \\ 1 & \text { otherwise }\end{cases}
$$

where $t_{b}$ is an empirically estimated threshold.

A first approximation of the height of the ridges $R$ and valleys $V$ is computed as

$$
\begin{aligned}
& R(x, y)=\log (G(x, y) \times(\neg B(x, y))), \\
& V(x, y)=\log (1-G(x, y) \times B(x, y)) .
\end{aligned}
$$

The intensity values of the ridges $R$ are then normalized in a range from $\left(1-\Delta_{r}\right)$ to 1 , and the intensity values of the valleys $V$ are then normalized in a range from 0 to $\Delta_{r}$. The value $\Delta_{r}$ was estimated by observing the shape of real threedimensional fingerprint models.

Then, the image $H$ describing the height of the ridge pattern in a non-dimensional space is computed as

$$
H(x, y)=R(x, y)+V(x, y) .
$$

As a last step, an adaptive histogram equalization is applied to $H$.

\section{Noise injection}

This step has the purpose of simulating the presence of pores, incipient ridges, and acquisition noise.

A binary image $B_{n}$, composed by randomly distributed white dots, is first computed. A morphological dilation with a mask of $n_{n} \times n_{n}$ pixel is then applied to $B_{n}$. Finally, the image $B_{n}$ is used to simulate pores and incipient ridges, using the equation:

$$
\begin{aligned}
H_{n}(x, y)= & H(x, y)-\Delta_{n}\left(B_{n}(x, y)(\neg B(x, y))\right) \\
& +\Delta_{N}\left(B_{n}(x, y) B(x, y)\right)
\end{aligned}
$$

where $H_{n}(x, y)$ is the resulting image and $\Delta_{n}$ is an empirically estimated value.

\section{Computation of the three-dimensional ridges}

In order to simulate the wear of the ridges, the height image $H_{n}$ is smoothed using an approach based on an empirically estimated threshold $t_{w}$. The value of $t_{w}$ corresponds to the $p$ percentile of the histogram of $H_{n}$. The ridge shape is obtained as:

$$
H_{q}(x, y)=\left\{\begin{array}{cl}
t_{w} & \text { if } H_{n}(x, y)>t_{w} \\
H_{n}(x, y) & \text { if } H_{n}(x, y) \leq t_{w}
\end{array} .\right.
$$

The height of the ridges in the image $H_{q}$ is then adjusted in order to have a mean value of $0.06 \mathrm{~mm}$. 


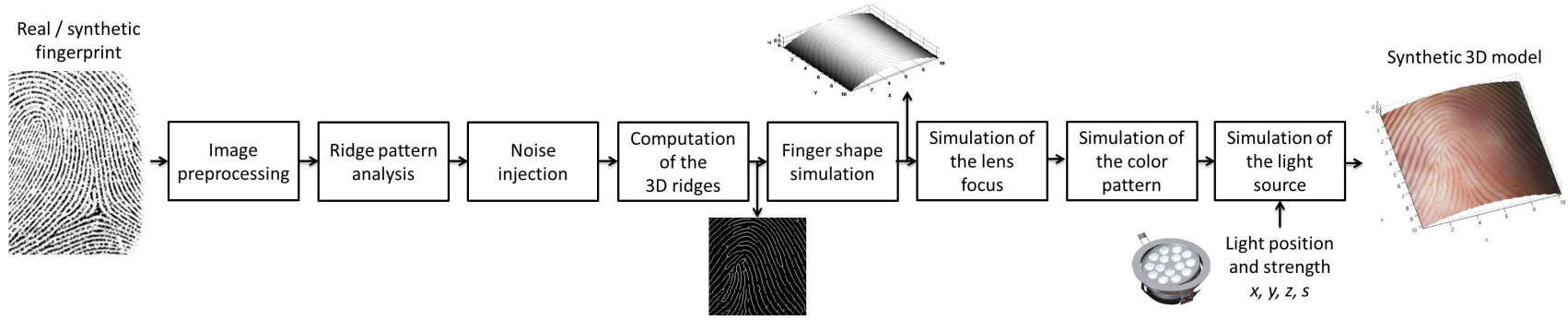

Fig. 2. Outline of the proposed approach for the computation of realistic three-dimensional synthetic fingerprints starting from contact-based fingerprint images.

\section{E. Finger shape simulation}

A parametric finger shape model based on a cylinder is computed:

$$
S(x, y)=\frac{-x^{2}+f_{n}}{f_{n}},
$$

where $S(x, y)$ is the three-dimensional surface based on the parametric model, and $f_{n}$ is a normalization term used to compute a surface with a peak height of $1 \mathrm{~mm}$. The peak height was experimentally estimated on an area of $5 \times 5 \mathrm{~mm}$ by considering the average curvature of the finger in its central area. The rendering of wider areas requires the usage of a better model of the finger surface, which is for example obtainable by using multiple view three-dimensional systems [4] or laser profilometers.

The normal vectors to each point of the surface $S(x, y)$ are then computed. The obtained result is a three-element vector $\left(N_{x}, N_{y}, N_{z}\right)$. The parametric surface is then converted to a full three-dimensional point cloud $\left(x_{s}, y_{s}, z_{s}\right)$, where $Z_{s}=$ $S(x, y)$.

The ridges are superimposed on the surface according to the direction of the normal to the surface:

$$
\begin{aligned}
& x_{r}=x_{s}+N_{x} H_{q}(x, y) \\
& y_{r}=y_{s}+N_{y} H_{q}(x, y) \\
& z_{r}=z_{s}+N_{z} H_{q}(x, y),
\end{aligned}
$$

where $H_{q}$ is the image describing the three-dimensional ridge structure. A surface approximation $S_{r}(x, y)$ is then computed from the point cloud $\left(x_{r}, y_{r}, z_{r}\right)$ using a linear interpolation technique.

\section{F. Simulation of the lens focus}

Real contactless fingerprint images can present regions affected by out of focus problems. In most of the cases, in fact, the hardware setups use macro lenses in order to capture the details of the ridge pattern. We then use a technique for the simulation of the lens focus in order to improve the realism of the obtained three-dimensional models. The proposed technique blurs every point of the ridge pattern proportionally to its height along the $z$ axis.

The height of every point $Z_{r}(x, y)$ is normalized between two empirically estimated values $b_{\min }$ and $b_{\max }$, obtaining the matrix $B_{s}$. For each point $(x, y)$, a median filter with a mask of $B_{s}(x, y) \times B_{s}(x, y)$ pixels, centered in $(x, y)$, is applied to $Z_{r}(x, y)$.

\section{G. Simulation of the color pattern}

The color pattern is acquired from a real contactless fingerprint image (Fig. 1). In this paper, we used contactless fingerprint samples captured using a technique similar to the one described in [4].

The ridge pattern is then removed from the contactless fingerprint image using a Gaussian low-pass filter, in order to extract only the underlying skin color and the vein pattern:

$$
I_{f}=I_{l} * G_{l},
$$

where $I_{f}$ is the resulting filtered image, $I_{l}$ is the contactless image, $G_{l}$ is a Gaussian $m \times m$ filter mask, with $\sigma$ equal to an empirically estimated value $\sigma_{p}$.

In order to improve the realism of the color pattern, speckle noise is introduced by applying the equation:

$$
I_{p}=I_{f}+n_{s} \times I_{f} * G_{l}
$$

where $n_{s}$ is uniformly distributed random noise with 0 mean and variance $v_{s}$. The value of $v_{s}$ is empirically estimated and is related to the simulated camera setup.

The image $I_{p}$ is then superimposed on the surface $S_{r}(x, y)$ using a texture mapping procedure.

\section{H. Simulation of the light source}

A punctiform light source with strength $s$, radiating uniformly in all directions, is then positioned at coordinates $\left(x_{l}, y_{l}, z_{l}\right)$ in order to simulate the illumination. The specular properties of the model are experimentally adjusted in order to meet the properties of the human skin. A Phong lighting algorithm is then used to compute the reflection model of the surface, but more complex rendering engines can be also applied [14].

Some examples of contact-based acquisitions and the resulting three-dimensional synthetic fingerprints are shown in Fig. 3.

\section{EXPERIMENTAL RESUltS}

In order to evaluate the proposed method, we acquired samples from 10 fingers. We captured 5 images for each fingerprint using a Crossmatch V300 contact-based sensor [15], and 5 images for each fingerprint using a Sony XCDSX90CR camera, using an acquisition setup similar to the one presented in [4]. The size of each contactless fingerprint image is $1280 \times 960$ pixel. We applied the described approach on the contact-based fingerprint images and compared the results 
(a)

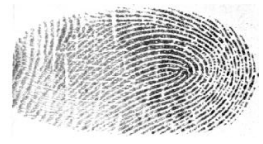

(d)

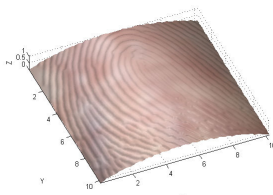

(b)

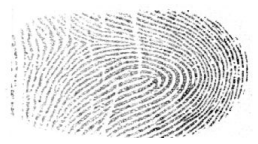

(e)

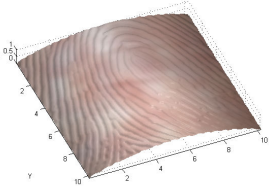

(c)

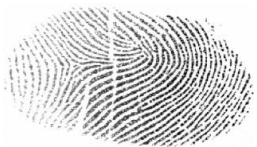

(f)

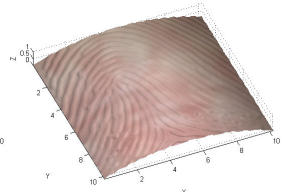

Fig. 3. Examples of synthetic three-dimensional fingerprints: (a-c) contactbased acquisitions; (d-f) three-dimensional synthetic fingerprints.

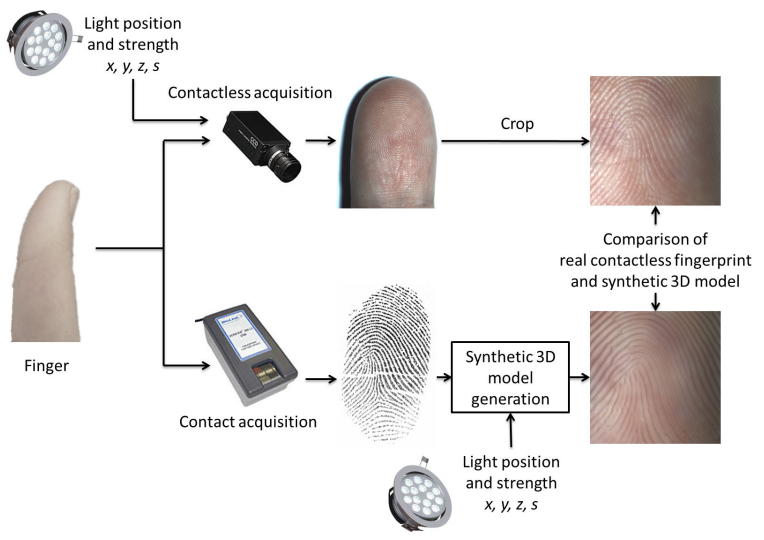

Fig. 4. Schema of the proposed evaluation method.

with the corresponding contactless fingerprint images. The parameters used in these experiments are: $t_{b}=0.1, \Delta_{r}=0.2$, $n_{n}=3, \Delta_{n}=0.2, t_{w}=60, b_{\min }=1, b_{\max }=3, m=30$, $v_{s}=0.0001, \sigma_{p}=0.7$.

The schema of the proposed evaluation method is shown in Fig. 4. We considered the parameters of the light illuminating the finger in the acquisition of the images, in particular the position and strength of the light, and simulate the same parameters in the computation of the synthetic fingerprint models by moving the light source across a reference Cartesian system centered on the three-dimensional model. The strength of the light source is also experimentally chosen in order to match the illumination used during the contactless acquisitions.

Examples of contactless fingerprint acquisitions and the corresponding synthetic three-dimensional models are shown in Fig. 5. In order to better evaluate the results, the corresponding central regions of the fingerprint images were cropped. From the images shown in Fig. 5, it is possible to observe that the proposed method achieves a realistic three-dimensional simulation of fingerprints, which resemble the corresponding contactless images. However, some regions of the obtained fingerprint models (in particular the external regions) present small differences compared to the corresponding contactless images. These differences are due to the fact that the proposed method estimates the height and the focus blur of the model by assuming a cylindrical approximating shape for the finger.
This assumption is reasonable in most of the cases, but can produce some small differences with respect to real contactless fingerprint images. Better results should be obtained by applying more complex models of the finger shape.

In order to evaluate the realism of the light source simulation, we also collected a set a of contactless fingerprints under different illumination conditions and used the proposed method to compute realistic synthetic models. The results obtained by simulating the light conditions shown in Fig. 1 are depicted in Fig. 6. In particular, we compared the synthetic models with real contactless images captured with an illumination from the left side (Fig. 6d), from the right side (Fig. 6e), and from the top side (Fig. 6f). It is possible to observe that the simulated fingerprint models are very similar to the corresponding contactless images.

We also tested the capability of the proposed method of simulating fingerprint models obtained using different kinds of cameras. As an example, Fig. 7 shows a comparison of the results obtained by simulating a fingerprint captured by a Sony XCD-SX90CR camera (Fig. 7a) and a VGA webcam (Fig. 7b). In order to compute the image shown in Fig. 7b, we used different parameters for the simulation of the lens focus $\left(b_{\min }=3, b_{\max }=5\right)$ and simulation of the color pattern $\left(v_{s}=0.001\right)$. It is possible to observe that the fingerprint model depicted in Fig. $7 \mathrm{~b}$ presents typical characteristics of the images captured using webcams, such as blur, out of focus regions, and noise.

\section{CONCLUSIONS}

In this paper, we proposed a novel technique for the simulation of synthetic three-dimensional models of fingerprints. The proposed technique can reduce the efforts needed to collect the data necessary for the validation of new algorithms operating on contactless fingerprint acquisitions. Moreover, the method can be used to compute a dataset of synthetic threedimensional models usable for comparing the performances of biometric algorithms. The proposed technique can then be useful for the entire research community working in the fields of biometrics and three-dimensional reconstruction.

The proposed method is based on image processing techniques and permits to simulate contactless fingerprint acquisitions performed in different light conditions by using different hardware setups. The method starts from a simulated fingerprint image or a real fingerprint image captured using a contact-based sensor. Well-known algorithms designed for fingerprint recognition systems are applied to the input image in order to extract the distinctive pattern of the ridges. Then, realistic effects such as noise, pores, and incipient ridges are introduced. The next step is the estimation of the threedimensional structure of the ridges, which is then superimposed on a parametric model of the finger shape, computed considering experimental measurements of the average finger curvature. In order to improve the realism of the simulated data, the lens focus blur is simulated. The model is then completed with the estimation of a realistic color pattern, obtained by applying a low-pass filter to a real contactless 
(a)

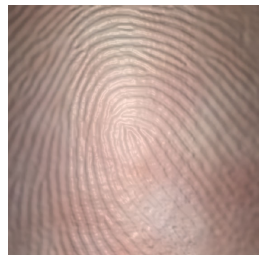

(f)

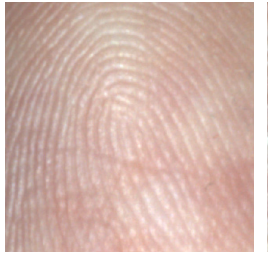

(b)

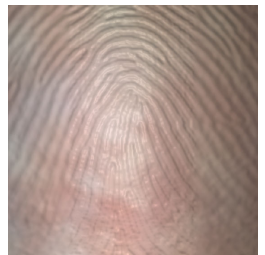

(g)

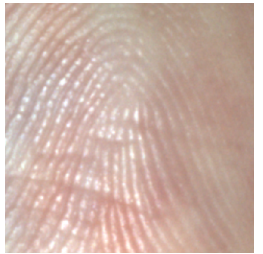

(c)

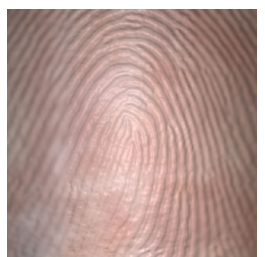

(h)

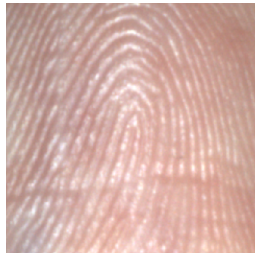

(d)

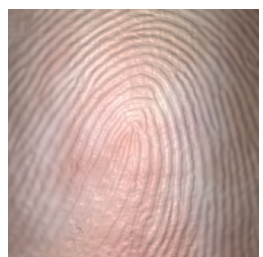

(i)

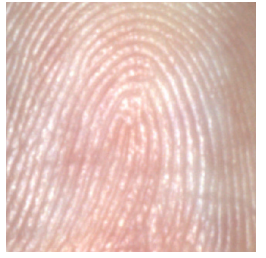

(e)

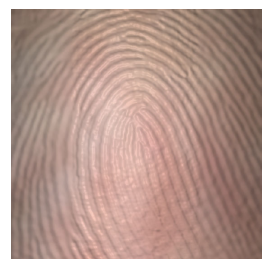

(j)

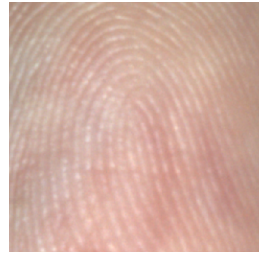

Fig. 5. Examples of the results of the proposed approach: (a-e) synthetic models computed using our approach; (f-j) corresponding contactless images.

(a)

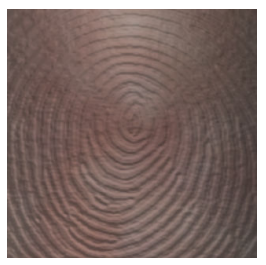

(d)

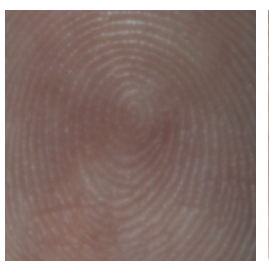

(b)

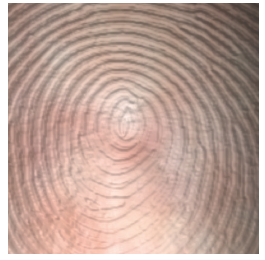

(e)

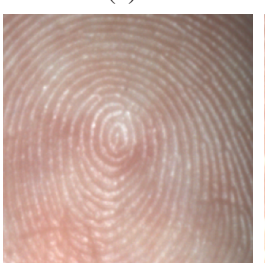

(c)

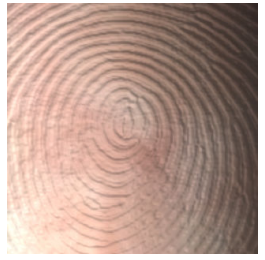

(f)

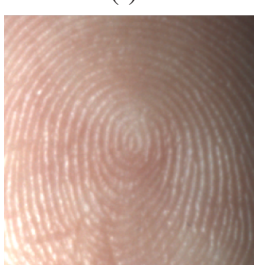

Fig. 6. Examples of the results of the proposed approach for different illumination conditions: (a-c) synthetic models obtained using our approach; (d-f) corresponding contactless images. The images $(\mathrm{a}, \mathrm{d})$ are related to an illumination from the left side; $(b, e)$ from the right side; $(c, f)$ from the top side.

fingerprint image, and by adding the properties of reflectance that match the ones of the human skin. Finally, a virtual light source is used to illuminate the scene and make the details of the ridges visible.

The obtained results show that the proposed method achieves a realistic modeling of the finger skin and its interaction with the light coming from the environment and/or the illumination system.

\section{REFERENCES}

[1] G. Parziale and Y. Chen, "Advanced technologies for touchless fingerprint recognition," in Handbook of Remote Biometrics, ser. Advances in Pattern Recognition, M. Tistarelli, S. Z. Li, and R. Chellappa, Eds. Springer London, 2009, pp. 83-109.

[2] G. Parziale, E. Diaz-Santana, and R. Hauke, “The surround imager: A multi-camera touchless device to acquire $3 \mathrm{D}$ rolled-equivalent fingerprints." in ICB'06, 2006, pp. 244-250.

[3] Y. Wang, L. Hassebrook, and D. Lau, "Data acquisition and processing of 3-D fingerprints," IEEE Transactions on Information Forensics and Security, vol. 5, no. 4, pp. 750-760, December 2010.

[4] R. Donida Labati, A. Genovese, V. Piuri, and F. Scotti, "Fast 3D fingertip reconstruction using a single two-view structured light acquisition," in 2011 IEEE Workshop on Biometric Measurements and

(a)

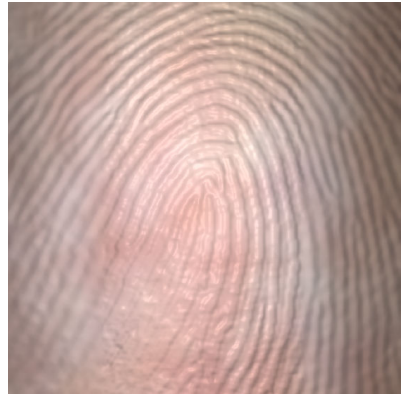

(b)

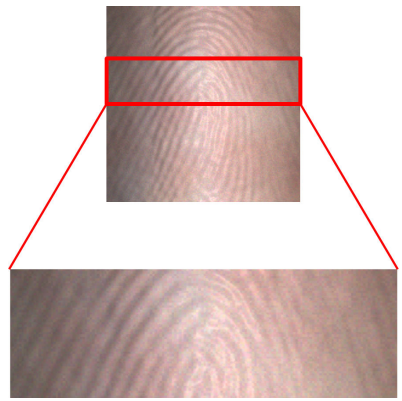

Fig. 7. Examples of results obtained by simulating a fingerprint captured by a Sony XCD-SX90CR camera (a) and a VGA webcam (b).

Systems for Security and Medical Applications (BIOMS), September 2011, pp. 1-8.

[5] J. Araque, M. Baena, B. Chalela, D. Navarro, and P. Vizcaya, "Synthesis of fingerprint images," in 16th International Conference on Pattern Recognition, vol. 2, 2002, pp. 422-425.

[6] M. Kücken and A. C. Newell, "A model for fingerprint formation," EPL (Europhysics Letters), vol. 68, no. 1, p. 141, 2004.

[7] M. Kücken, "Models for fingerprint pattern formation," Forensic Science International, vol. 171, no. 2-3, pp. 85-96, 2007.

[8] U.-K. Cho, J.-H. Hong, and S.-B. Cho, "Automatic fingerprints image generation using evolutionary algorithm," in Proceedings of the 20th international conference on Industrial, engineering, and other applications of applied intelligent systems, ser. IEA/AIE'07. Berlin, Heidelberg: Springer-Verlag, 2007, pp. 444-453.

[9] R. Cappelli, "Synthetic fingerprint generation," in Handbook of Fingerprint Recognition, 2nd ed., A. J. D. Maltoni, D. Maio and S. Prabhakar, Eds. Springer London, 2009, pp. 271-301.

[10] J. Xue, S. Xing, Y. Guo, and Z. Liu, "Fingerprint generation method based on gabor filter," in 2010 International Conference on Computer Application and System Modeling (ICCASM), vol. 8, October 2010, pp. 115-119.

[11] S. Ferrari, V. Piuri, and F. Scotti, "Virtual environment for granulometry analysis," in IEEE International Conference on Virtual Environments, Human-Computer Interfaces and Measurement Systems (VECIMS), July 2008, pp. 156-161.

[12] A. Genovese, R. Donida Labati, V. Piuri, and F. Scotti, "Virtual environment for synthetic smoke clouds generation," in IEEE International Conference on Virtual Environments, Human-Computer Interfaces and Measurement Systems (VECIMS 2011), September 2011, pp. 1-6.

[13] P. D. Kovesi, "MATLAB and Octave functions for computer vision and image processing," Centre for Exploration Targeting, School of Earth and Environment, The University of Western Australia, available from: $<$ http://www.csse.uwa.edu.au/ pk/research/matlabfns/ $>$.

[14] Blender, http://www.blender.org/.

[15] Cross Match Technologies, Inc., http://www.crossmatch.com/. 